\title{
Nutrition, Genes, and Age-Related Macular Degeneration: What Have We Learned from the Trials?
}

\author{
Emily Y. Chew \\ National Eye Institute, National Institutes of Health, Bethesda, MD, USA
}

\section{Keywords}

Age-related macular degeneration - Clinical trials - Genetics

\begin{abstract}
The Age-Related Eye Disease Study (AREDS) and AREDS2 provided evidence for treating persons with age-related macular degeneration (AMD) with antioxidant vitamins and minerals to reduce the risk of development of late AMD. The AREDS2 data suggest that the beta-carotene in the original AREDS supplements be replaced by lutein and zeaxanthin, providing a safer drug for those who are smokers or former smokers. Even though consuming fish reduced the risk of AMD in observational studies, the AREDS2 results showed that omega-3 long-chain polyunsaturated fatty acids (docosahexaenoic acid/eicosapentaenoic acid) had no beneficial effect on AMD. Despite the major progress in the discovery of gene variants associated with $A M D$, the use of genetic testing to predict disease has not been clinically useful. The use of genetic testing prior to AMD therapies such as administering AREDS supplements is not recommended by the American Academy of Ophthalmology and other organizations.
\end{abstract}

(C) 2017 S. Karger AG, Basel

\section{Introduction}

It is my greatest privilege and honor to deliver the 2016 Ingrid Kreissig Lecture in the beautiful city of Copenhagen at the exciting EURORETINA meeting. I thank Professor Kreissig, the committee, and the organizers for this tremendous honor. I am very humbled to join a list of luminaries who are former Ingrid Kreissig lecturers. As many of you know, Professor Ingrid Kreissig is a role model to many of us, including young women aspiring to be clinician-scientists. She has authored hundreds of papers and many, many textbooks. She is indeed the master of retinal detachment surgery.

Professor Kreissig has directed an academic ophthalmology program and trained numerous ophthalmologists. Wherever she goes, she is surrounded by trainees and colleagues eager to learn from her. She is the mentor for mentors. She has trained several hundred ophthalmologists from all over the world. They have, in turn, become teachers and heads of departments of ophthalmology. The impact of her mentorship clearly grows exponentially.

She continues with her passion for teaching and is still actively conducting educational courses throughout the world. She is indeed a remarkable researcher, clinician, and teacher. "Professor" has made a meaningful impact in the field of ophthalmology through her generous spirit.

Emily Y. Chew, MD

National Eye Institute, National Institutes of Health, Building 10

CRC Room 3-2531, 10 Center Drive, MSC 1204

Bethesda, MD 20892-1204 (USA)

E-Mail echew@nei.nih.gov 


\section{Nutrition, Genes, and Age-Related Macular Degeneration}

I will now address the topic of my lecture: nutrition, genes and age-related macular degeneration (AMD). What have we learned from the recent trials? These study results are the culmination of the work conducted by many collaborators in the Age-Related Eye Disease Study (AREDS) and the AREDS2. I am grateful for their collaboration.

It is no doubt that we are indeed what we eat. The first clue that AMD may have association with dietary risk factors comes from the first National Health and Nutrition Examination Survey (NHANES) survey, which was published in 1988 [1]. Diets rich in fruits and vegetables with vitamins, especially vitamin $A$, were inversely associated with AMD. Since then, numerous studies have suggested the importance of dietary risk factors, and clinical trials were conducted to evaluate specific antioxidant vitamins and zinc.

\section{The AREDS and AREDS2}

The AREDS, a study supported by the National Institutes of Health, was designed to study the natural course of the age-related cataract and AMD. It also evaluated the nutritional risk factors, which began in 1992 as randomized controlled clinical trial assessing antioxidant vitamins C (500 mg), E (400 international units), and betacarotene $(15 \mathrm{mg})$, and zinc ( $80 \mathrm{mg}$ of zinc oxide) with copper ( $2 \mathrm{mg}$ of cupric oxide). This was a factorial design with 3,640 participants randomized to placebo $(n=903)$, antioxidants $(n=945)$, zinc $(n=904)$, and to the combination of antioxidants and zinc $(n=888)$. In 2001, after a median follow-up of 6.5 years, the results of the study showed that the AREDS supplements were beneficial in reducing the risk of progression to late AMD by $25 \%$ [2]. This AREDS formulation was found to have persistent beneficial effects with 10 years of follow-up [3].

Observational data from AREDS suggested that the increased dietary intake of lutein and zeaxanthin in green leafy vegetable such as kale, collard greens, and spinach, and omega-3 long-chain polyunsaturated fatty acids (LCPUFAs) found in fish were inversely associated with the risk of late AMD $[4,5]$. This was supported by other observational studies, such as population-based studies or other case-control studies [6-11].

Observational data cannot provide causal effects and can only determine associations. These associations may be due to other confounding factors. These suggested nu- trients must be studied in a randomized controlled clinical trial to evaluate their potential therapeutic effects on AMD.

AREDS2 was designed to evaluate the effects of lutein/ zeaxanthin and omega-3 LCPUFAs on AMD [12]. Because the AREDS2 participants were at high risk of developing late AMD, the AREDS formulation would be recommended to them. The treatment effect of the additional nutrients, lutein/zeaxanthin and omega-3 LCPUFAs was evaluated as the effect beyond that of AREDS supplement, which is known to reduce the risk of progression to late AMD. The primary goal of AREDS2 was to determine whether adding lutein/zeaxanthin and/or docosahexaenoic acid/eicosapentaenoic acid (DHA/EPA) to the AREDS formulation decreases the risk of late AMD, defined as neovascular AMD or central geographic atrophy. A secondary objective is to evaluate the effect of eliminating beta-carotene and/or lowering zinc levels in the AREDS formulation.

The AREDS2 was a multicenter phase 3 randomized, double-masked, placebo-controlled trial conducted between 2006 and 2012, which enrolled participants, aged 50-85 years, at risk for progression to AAMD. The primary randomization included the daily AREDS supplement plus one of the following: lutein/zeaxanthin (10/2 $\mathrm{mg})$, DHA/EPA (350/650 mg), a combination of these 2, or placebo. The secondary randomization consisted of the elimination of beta-carotene and/or lowering of zinc to $25 \mathrm{mg}$ instead of the original $80 \mathrm{mg}$ found in the AREDS formulation. Both were factorial designs. The major outcome measurement was the development of late AMD, either neovascular AMD or central geographic atrophy, as documented by centralized grading of annual retinal photographs and by treatment history.

\section{Results of the AREDS2}

In 82 academic and community centers across the United States, 4,203 participants with either bilateral large drusen or large drusen in 1 eye with late AMD in the fellow eye were enrolled. Population characteristics at baseline were as follows: mean (standard deviation) age 73.1 (7.7) years, $57 \%$ female, $97 \%$ white, $7 \%$ current smokers, $19 \%$ with prior cardiovascular disease, and 44 and $50 \%$ taking statin-class cholesterol-lowering drugs and aspirin, respectively. About two-thirds of the participants had bilateral large drusen or less severe, and one-third had advanced AMD in 1 eye and a mean visual acuity of 20/32 in eyes without advanced AMD. They were followed for a median of 4.9 years (interquartile range: 4.3 ,
2
Ophthalmologica 2017;238:1-5 DOI: $10.1159 / 000473865$
Chew 
5.1). Three percent were lost to follow-up, and $9 \%$ died. Compliance was good with $85 \%$ of the participants taking $75 \%$ of their study supplements.

The primary analyses compared each of the treatment groups with placebo, and there were no statistically significant differences [13]. However, when using the entire population in the evaluation comparing lutein/zeaxanthin versus no lutein/zeaxanthin in the so-called main effects analyses that doubled the sample size, there was a $10 \%$ reduction in the risk developing advanced AMD (hazard ratio [HR]: 0.90, 95\% confidence interval [CI]: $0.82-0.99, p=0.04)$. When the effect was evaluated, stratified by the different levels of dietary intake of lutein/zeaxanthin, the HR was 0.74 with a $95 \%$ CI of $0.59-0.94, p=$ 0.01 , for those in the lowest level of dietary intake, in favor of supplementation with lutein/zeaxanthin. When tested for the 2 separate outcomes of late AMD, the HRs were 0.89 (CI: $0.79-1.00, p=0.05$ ) for the development of neovascular AMD and 0.92 (CI: 0.78-1.07, $p=0.27$ ) for development of central geographic atrophy. Further secondary analyses were conducted on lutein/zeaxanthin [14]. We conducted a head-to-head comparison of lutein/ zeaxanthin versus beta-carotene by comparing those randomized to lutein/zeaxanthin and the AREDS formulation minus the beta-carotene with those randomized to the AREDS formulation with beta-carotene (and NO lutein/zeaxanthin). The HRs for lutein/zeaxanthin versus beta-carotene comparisons were 0.82 (95\% CI: 0.69$0.96), p=0.02$ for progression to late AMD; 0.78 (95\% CI: $0.64-0.94, p=0.01)$ for neovascular AMD, and 0.94 (95\% CI: $0.70-1.26), p=0.67$ for central geographic atrophy.

In analyses restricted to those with bilateral large drusen at baseline, the HRs for lutein/zeaxanthin versus no lutein/zeaxanthin were 0.87 (95\% CI: 0.77-0.95, $p=0.04$ ) for developing late AMD; 0.80 (95\% CI: 0.68-0.95, $p=$ $0.01)$ for progression to neovascular AMD, and 0.94 ( $95 \%$ CI: $0.78-1.13, p=0.51$ ) for progression to central geographic atrophy.

Another interesting analysis was the comparison of lutein/zeaxanthin plus beta-carotene versus beta-carotene alone, showing a HR of 0.82 (95\% CI: 0.69-0.97, $p=0.02$ ) for the development of late AMD. This same analysis for the development of neovascular AMD showed a HR of 0.72 (95\% CI: $0.59-0.89, p=0.002$ ). This suggests that the effects are driven by lutein/zeaxanthin and that beta-carotene has a minimal role.

Individuals randomized to beta-carotene had double the risk of lung cancer ( 2 vs. $0.9 \%$ ), $p=0.04 ; 91 \%$ of the incident lung cancers were in former smokers. This suggests that beta-carotene has evidence of harmful effects in

Nutrition, Genes, and AMD past smokers. For these reasons, the AREDS2 supplements, which replaced the beta-carotene with lutein and zeaxanthin, is preferred.

The main effects analyses of low zinc ( $25 \mathrm{mg}$ as zinc oxide) versus high zinc ( $80 \mathrm{mg}$ as zinc oxide) showed no difference in the primary outcome of progression to late AMD or adverse effects. These were not considered noninferiority analyses as we were not sufficiently powered or designed for such analyses. Therefore, we were not able to conclude that the 2 doses be similar in effect. Because of data from the original AREDS, which was a placebocontrolled trial demonstrating the beneficial effects of 80 $\mathrm{mg}$ of zinc, it was decided that AREDS2 supplement would continue to maintain $80 \mathrm{mg}$ of zinc.

\section{AREDS2 Cardiovascular Outcome Study}

The AREDS Cardiovascular Outcome Study (COS) [15] was an ancillary study designed to evaluate the role of supplementation with DHA/EPA $(350 / 650 \mathrm{mg})$ or lutein/zeaxanthin $(10 / 2 \mathrm{mg})$ in reducing the risk of cardiovascular disease. The primary outcome was a composite outcome of myocardial infarction, stroke, and cardiovascular death. We also had prespecified secondary combinations of the primary outcome with hospitalized heart failure, revascularization, or unstable angina.

The results showed that a total of 602 cardiovascular events were adjudicated by an expert panel, and 459 were found to meet the criteria that were defined as an outcome. Using an intention-to-treat analysis, there was no reduction in the risk of cardiovascular disease or secondary cardiovascular outcomes for DHA plus EPA with HR: 0.95, 95\% CI: $0.78-1.17$ or for lutein/zeaxanthin with HR $0.94,95 \%$ CI: $0.77-1.15$. There were no differences in the adverse effects by treatment group. We had $80 \%$ power to detect a $25 \%$ reduction in the cardiovascular events.

We concluded that despite numerous epidemiologic evidence of the beneficial effect of consuming fish, oral supplementation of DHA and EPA or lutein/zeaxanthin did not reduce the risk of cardiovascular disease in these participants with evidence of AMD.

\section{AREDS2 Cognitive Function Study}

We also evaluated the role of oral supplements with DHA plus EPA and lutein/zeaxanthin for cognitive function in the AREDS2 population [16] as epidemiologic data suggested that high dietary intake of saturated fat and low

Ophthalmologica 2017;238:1-5 DOI: $10.1159 / 000473865$ 
intake of fruits and vegetables may be associated with an increased risk of impaired cognitive function. Several validated cognitive function tests were administered to the AREDS2 participants by telephone by certified examiners at baseline and every 2 years during the 5 -year study. The primary outcome of this AREDS2 ancillary study was the yearly change in the composite score determined from the battery of cognitive function test from baseline. While adjusted for baseline age, race, sex, hypertension, education, cognitive score, and depression score, we analyzed the differences in the composite scores between the treated versus the untreated groups in AREDS2.

The results of the 3,741/2,403 (89\%) of the AREDS2 participants who enrolled in this ancillary study showed no statistically significant differences in the change of scores for the participants assigned to receive the supplements versus those who were assigned to not receive the supplements. The changes in composite cognitive function score ranged from -22 to 17 , with the better cognitive function represented by the high numeric score. The yearly change in the composite cognitive function score was -0.19 (99\% CI: -0.25 to -0.13 ) for the AREDS2 participants assigned to receive $\mathrm{DHA}+\mathrm{EPA}$ versus -0.18 (99\% CI: -0.24 to -0.12 ) for those assigned to no DHA+EPA. The yearly change between the 2 treatment groups were not statistically significantly different (difference in yearly change, -0.03 [99\% CI: -0.20 to 0.13], $p=0.63$ ). The same was true for the lutein/zeaxanthin treatment arm as well. For those randomized to receive lutein/zeaxanthin, the yearly change in the composite cognitive function score was -0.18 (99\% CI: -0.24 to -0.11 ) versus -0.19 (99\% CI: -0.25 to -0.13 ) for those randomized to no lutein/zeaxanthin (difference in yearly change, 0.03 [99\% CI: -0.14 to 0.19 ], $p=0.66$ ). We concluded that despite the epidemiologic data support, the beneficial role of consuming omega- 3 fatty acids in fish, oral supplementation with DHA+EPA, and lutein/zeaxanthin in persons with at least intermediate AMD did not have a beneficial or harmful effect.

\section{Genetic Testing prior to Supplementing with AREDS2 Supplements}

Previous reports suggested that genetic testing for AMD may have a role because of potential harmful genetic interaction with specifically the zinc component in the AREDS supplements $[17,18]$. Following the publication of differing opinions [19], other genetic, statistical, and epidemiologic experts $[20,21]$ and a panel of experts from the American Society of Retinal Surgeons (ASRS) weighed in [22]. They all supported the recommendations of the American Academy of Ophthalmology that genetic testing for AMD should not be conducted prior to considering treatments for AMD such as the AREDS supplements [23].

\section{Summary}

The results of the epidemiologic and clinical trials have pointed to the importance of nutritional factors in AMD, cardiovascular disease, and cognitive function. Although the clinical trials did not show a beneficial effect of supplementation with omega-3 fatty acids in these 3 diseases, eating fish seemed to be important in many epidemiologic studies. In addition to maintaining a healthy lifestyle of weight control and eliminating tobacco use, persons at risk for AMD or who have intermediate AMD or worse should consider a diet replete with fish and green leafy vegetables. For potential beneficial effects, fish may be important for cardiovascular disease as well as cognitive function. The AREDS and AREDS2 supplements are also recommended for persons with at least intermediate AMD or late AMD in 1 eye. AREDS2 is safe, especially for smokers and former smokers who are at risk for developing lung cancer. Removing beta-carotene has reduced that risk. Genetic testing prior to AMD treatments, especially with AREDS/AREDS2 supplements, is not recommended by the American Academy of Ophthalmology and other organizations.

\section{Acknowledgements}

The AREDS2 clinical trial was supported by the intramural program funds and contracts from the National Eye Institute/National Institutes of Health, Department of Health and Human Services, Bethesda, MD, USA (contract HHS-N-260-2005-00007-C; ADB contract NO1-EY-5-0007). Funds were generously contributed to these contracts by the following NIH institutes: Office of Dietary Supplements, National Center for Complementary and Alternative Medicine; National Institute on Aging; National Heart, Lung, and Blood Institute, and National Institute of Neurological Disorders and Stroke.

\section{Disclosure Statement}

Emily Chew is an employee of the National Eye Institute/National Institutes of Health and is supported by the intramural program. While Emily Chew has no personal financial disclosures, the National Institutes of Health holds a royal bearing license issued to Bausch and Lomb for the Age-Related Eye Disease Study (AREDS) Supplement.
4

Ophthalmologica 2017;238:1-5 DOI: $10.1159 / 000473865$
Chew 


\section{References}

1 Goldberg J, Flowerdew G, Smith E, Brody JA, Tso MO: Factors associated with age-related macular degeneration. An analysis of data from the first National Health and Nutrition Examination Survey. Am J Epidemiol 1988; 128:700-710.

2 The Age-Related Eye Disease Study Research Group: A randomized, placebo-controlled, clinical trial of high-dose supplementation with vitamins $\mathrm{C}$ and $\mathrm{E}$, beta carotene, and zinc for age-related macular degeneration and vision loss: AREDS report No. 8. Arch Ophthalmol 2001;119:1417-1436.

3 Chew EY, Clemons TE, Agrón E, Sperduto RD, Sangiovanni JP, Kurinij N, Davis MD; Age-Related Eye Disease Study Research Group: Long-term effects of vitamins $\mathrm{C}$ and $\mathrm{E}, \beta$-carotene, and zinc on age-related macular degeneration: AREDS report No. 35. Ophthalmology 2013;120:1604-1611.

4 SanGiovanni JP, Chew EY, Clemons TE, Ferris FL 3rd, Gensler G, Lindblad AS, Milton RC, Seddon JM, Sperduto RD; and the AgeRelated Eye Disease Study Research Group: The relationship of dietary carotenoids, vitamin $\mathrm{E}$, and vitamin $\mathrm{C}$ with age-related macular degeneration: a case-control study in the Age-Related Eye Disease Study: AREDS report No. 22. Arch Ophthalmol 2007;125: $1225-1232$

5 SanGiovanni JP, Agrón E, Meleth AD, Reed GF, Sperduto RD, Clemons TE, Chew EY: Omega-3 long-chain polyunsaturated fatty acid intake and 12-year incidence of neovascular age-related macular degeneration and central geographic atrophy: a prospective cohort study from the Age-Related Eye Disease Study. Am J Clin Nutr 2009;90:1601-1607.

6 Seddon JM, Ajani UA, Sperduto RD, Hiller R, Blair N, Burton TC, Faber MD, Gragoudas ES, Haller J, Miller DT, et al: Dietary carotenoids, vitamins $\mathrm{A}, \mathrm{C}$, and $\mathrm{E}$, and advanced age-related macular degeneration. JAMA 1994;272:1413-1420.

7 Augood C, Chakravarthy U, Young I, Vioque J, de Jong PT, Bentham G, Rahu M, Seland J, Soubrane G, Tomazzoli L, Topouzis F, Vingerling JR, Fletcher AE: Oily fish consumption, dietary docosahexaenoic acid and eicosapentaenoic acid intakes, and associations with neovascular age-related macular degeneration. Am J Clin Nutr 2008;88:398406.
8 Mares-Perlman JA, Fisher AI, Klein R, Palta M, Block G, Millen AE, Wright JD: Lutein and zeaxanthin in the diet and serum and their relation to age-related maculopathy in the third national health and nutrition examination survey. Am J Epidemiol 2001;153:424-432.

9 Moeller SM, Parekh N, Tinker L, Ritenbaugh C, Blodi B, Wallace RB, Mares JA; CAREDS Research Study Group: Associations between intermediate age related macular degeneration and lutein and zeaxanthin in the Carotenoids in Age-Related Eye Disease Study (CAREDS): ancillary study of the Women's Health Initiative. Arch Ophthalmol 2006;124: 1151-1162.

10 Tan JS, Wang JJ, Flood V, Rochtchina E, Smith W, Mitchell P: Dietary antioxidants and the longer-term incidence of age-related macular degeneration: the Blue-Mountains Eye Study. Ophthalmol 2008;115:334-341.

11 Seddon JM, Cote J, Rosner B: Progression of age-related macular degeneration: association with dietary fat, transunsaturated fat, nuts, and fish intake. Arch Ophthalmol 2003; 121:1728-1737.

12 The AREDS2 Research Group, Chew EY, Clemons T, SanGiovanni JP, Danis RP, Domalpally A, McBee W, Sperduto R, Ferris FL: The Age-Related Eye Disease Study 2 (AREDS2): study design and baseline characteristics (AREDS2 report number 1). Ophthalmology 2012;119:2282-2289.

13 Age-Related Eye Disease Study 2 Research Group: Lutein + zeaxanthin and omega-3 fatty acids for age-related macular degeneration: the Age-Related Eye Disease Study 2 (AREDS2) randomized clinical trial. JAMA 2013;309:2005-2015.

14 The Age-Related Eye Disease Study 2 (AREDS2) Research Group, Chew EY, Clemons TE, SanGiovanni JP, Danis RP, Ferris FL 3rd, Elman MJ, Antoszyk AN, Ruby AJ, Orth D, Bressler SB, Fish GE, Hubbard GB, Klein ML, Chandra SR, Blodi BA, Domalpally A, Friberg T, Wong WT, Rosenfeld PJ, Agrón E, Toth CA, Bernstein PS, Sperduto RD: Secondary analyses of the effects of lutein/zeaxanthin on age-related macular degeneration progression: AREDS report No. 3. JAMA Ophthalmol 2014;132:142-149.
15 Bonds DE, Harrington M, Worrall BB, Bertoni AG, Eaton CB, Hsai J, Robinson J, Clemons TE, Fine LJ, Chew EY: Effect of longchain omega-3 fatty acids and lutein + zeaxanthin supplements on cardiovascular outcomes: results of the Age-Related Eye Disease Study 2 (AREDS2) randomized clinical trial. JAMA Intern Med 2014;714:783-771.

16 Chew EY, Clemons TE, Agron E, Launer L, Grodstein F, Berstein PS; Age-Related Eye Disease Study 2 (AREDS2) Research Group: Effect of omega-3 fatty acids, lutein/zeaxanthin, or other nutrient supplementation on cognitive function: The AREDS2 randomized clinical trial. JAMA 2015;314:791-801.

17 Awh CC, Lane AM, Hawken S, Zanke B, Kim IK: CFH and ARMS2 genetic polymorphisms predict response to antioxidants and zinc in patients with age-related macular degeneration. Ophthalmology 2013;120:2317-2323.

18 Awh CC, Hawken S, Zanke BW: Treatment response to antioxidants and zinc based on $\mathrm{CFH}$ and ARMS2 genetic risk allele number in the Age-Related Eye Disease Study. Ophthalmology 2015;122;162-169.

19 Chew EY, Klein ML, Clemons TE, Agrón E, Ratnapriya R, Ewards AO, Fritsche LG, Swaroop A, Abecasis GR; the Age-Related Eye Disease Study: No clinically significant association between $\mathrm{CFH}$ and ARMS2 genotypes and response to nutritional supplements: AREDS report number 38. Ophthalmology 2014;121:2173-2180.

20 Stone EM: Genetic testing for age-related macular degeneration: not indicated now. JAMA Ophthalmol 2015;133:598-600

21 Wittes J, Musch DC: Should we test for genotype in deciding on Age-Related Eye Disease Study supplementation? Ophthalmology 2015;122:3-5

22 Csaky KG, Schachat AP, Kaiser PK, Small KW, Heier JS: The use of genetic testing in the management of patients with age-related macular degeneration: American Society of Retina Specialists Genetics Task Force Special Report. Journal of VitreoRetinal Diseases 2017;1:75-78.

23 Stone EM, Aldave AJ, Drack AV, MacCumber MW, Sheffield VD, Traboulsi E, Weleber RG: Recommendations for genetic testing of inherited eye diseases. Report of the American Academy of Ophthalmology Task Force on Genetic Testing. Ophthalmology 2012;119: 2408-2410.

Nutrition, Genes, and AMD

Ophthalmologica 2017;238:1-5 